\title{
Statistical analysis of nonlinear optical amplifier in high saturation
}

\author{
S. Ruiz-Moreno \\ G. Junyent \\ M.J. Soneira \\ J.R. Usandizaga
}

Indexing terms: Optical amplifiers, Quantum electronics, Mathematical techniques

\begin{abstract}
In the work, analytic expressions describing the behaviour of a strongly saturated nonlinear optical amplifier are deduced. These expressions make possible the calculation of the mean and variance of the amplified light photon number. Results are compared with those obtained by the numerical resolution of the photon density matrix equation, and good accordance is observed in the strong saturation regime. The results numerically obtained enable us to make conclusions about the statistical fluctuations of the optical power at the nonlinear optical amplifer output.
\end{abstract}

\section{Introduction}

One of the devices which has become more interesting in digital fibre optic transmission systems is the semiconductor optical amplifier $[1,2]$. By the direct amplification of light, its use makes possible the elimination of the double optoelectric and electro-optic conversion, reducing the number of electronic components and their associated problems. Nevertheless, owing to quantum noise, the optical amplifier modifies the statistical parameters of the incident optical power, giving rise to a degradation of the signal-to-noise ratio. The latter has been studied in several works $[1,3,4,5,6,11]$, assuming a linear behaviour of the device (absence of saturation). Actually, that assumption is inadequate in many cases because spontaneous emission as well as the power level of the input signal can lead the amplifier to a state of appreciable saturation. It is then necessary to solve the nonlinear equations characterising the device, to calculate the mean and variance of the amplified light. In Reference 7 , the authors describe a numerical method to solve these equations, taking into account the saturation phenomenon.

The behaviour of an optical amplifier can be considered to be linear when the stimulated emission and absorption probabilities are constant and independent of the incident optical power. We obtain the equations put forward by Shimoda, Takahasi and Townes [9], which do not take into account the possible decay of the energy

Paper 5865J (E13, E3), first received 9th June and in revised form 6th November 1987

The authors are with the Department of Signal Theory and Communications, ETSIT Barcelona, C/Jorge Girona Salgado s/n, 08034 Barcelona, Spain levels of the amplifying medium owing to the spontaneous emission. When the incident power is high, it is necessary to take into account this decay because the saturation phenomenon is appreciable and the amplifier has a nonlinear behaviour. On the other hand, the amplifier tends to behave linearly when it is strongly saturated [14].

In this paper it is shown that the behaviour of an optical amplifier in the case of very strong saturation is approximately linear. Analytical expressions for the mean and variance of the optical output power in this situation are obtained. Using the method described in Reference 7 , the nonlinear differential equations characterising the amplifier are numerically solved in order to confirm the validity of the analytic expressions in the very-strong saturation case. The accordance is good, as can be seen in the presented curves. On the other hand, the results obtained numerically show that the signal-to-noise ratio of the amplified optical power can be optimised theoretically as a function of the different parameters which take part.

\section{Theory}

The working principle of an optical amplifier is essentially the same of a laser diode. Its statistical behaviour can be characterised starting from the photon density matrix equation $[8,15]$ which, despite the fact that it does not take into account the noise owing to carrier fluctuations, describes the temporary evolution of the probability density function, $P_{m}(t)$, having $m$ photons in the considered mode at an instant $t$ when there are $n$ photons at the amplifer input $(t=0)$. According to this equation, the evolution of $P_{m}(t)$ during the amplification process can be expressed as

$$
\begin{aligned}
\frac{d P_{m}(t)}{d t}= & -\frac{(m+1) A}{1+(m+1) s} P_{m}(t)+\frac{m A}{1+m s} P_{m-1}(t) \\
& +\frac{(m+1) B}{1+(m+1) s} P_{m+1}(t)-\frac{m B}{1+m s} P_{m}(t) \\
& +(m+1) C P_{m+1}(t)-m C P_{m}(t)
\end{aligned}
$$

where $A$ and $B$ are coefficients proportional to the photon stimulated emission and absorption, respectively, $s$ is the saturation parameter which coincides with the spontaneous emission coefficient [8], and $C$ is a coefficient which represents the linear losses of the amplifying

IEE PROCEEDINGS, Vol. 135, Pt. J, No. I, FEBRUARY 1988 
medium. The values of $A, B$ and $C$ are given by

$$
\begin{aligned}
& A=\frac{n_{s p}}{z_{p}} \cdot \frac{P}{P_{t h}} \\
& B=\frac{n_{s p}-1}{z_{p}} \\
& C=\frac{1}{z_{P}}
\end{aligned}
$$

where $n_{s p}$ is the population inversion parameter, $P$ represents the pumping rate, with a threshold value $P_{t h}$, and $z_{p}$ is the photon lifetime.

Eqn. 1 takes into account, besides the linear losses of the amplifying medium, the saturation process of the stimulated emission as well as the saturation process of the stimulated absorption. Its linear version $(s=0)$ corresponds to the equation put forward by Shimoda et al. [9] for the quanta amplification. From eqn. 1 the nonlinear differential equations set (describing the temporary evolution of the moments of the photon distribution during the amplification process) can be obtained without difficulty. For the $r$ th order moment, which we will call $m_{r}$, it is easy to show that eqn. 1 leads to

$$
\begin{aligned}
\frac{d m_{r}}{d t}= & \sum_{j=1}^{r}\left(\begin{array}{c}
r \\
j-1
\end{array}\right)\left[\left\langle p_{j}(m)\right\rangle A\right. \\
& \left.+(-1)^{r+j-1}\left\langle q_{j}(m)\right\rangle B+(-1)^{r+j-1}\left\langle m^{j}\right\rangle C\right]
\end{aligned}
$$

where the symbol $\langle>$ expresses the statistical mean value. We have defined

$$
p_{j}(m)=\frac{(m+1) m^{j-1}}{1+(m+1) s}
$$

and

$$
q_{j}(m)=\frac{m^{j}}{1+m s}
$$

Eqn. 3 gives, for different values of $r$, a coupled nonlinear equations system. The numerical solution of these equations enables us to study the behaviour of the photon distribution moments as a function of the amplification time and of the saturation parameter value. In Section 4 we present the results obtained for the mean, the variance and the signal-to-noise ratio of the photon number at the amplifier output when there is an optical pulse at the input.

\section{Statistical model of the strongly saturated optical amplifier}

In an optical amplifier, the saturation phenomenon can be considered negligible when the value of the saturation parameter $s$ is such that $m s \ll 1$. The opposite case, i.e., the one that verifies $m s \gg 1$, is called 'situation of strong saturation'. Under these conditions, the functions defined in eqn. 4 are identical and can be approximated by

$$
p_{j}(m)=q_{j}(m) \simeq \frac{m^{j-1}}{s}
$$

and, then, eqn. 3 takes the following form

$$
\begin{aligned}
\frac{d m_{r}}{d t} \simeq & \sum_{j=1}^{r}\left(\begin{array}{c}
r \\
j-1
\end{array}\right)\left\{\frac{1}{s} m_{j-1}\left[A+B(-1)^{r+j-1}\right]\right. \\
& \left.+(-1)^{r+j-1} m_{j} C\right\}
\end{aligned}
$$

where $m_{j}=\left\langle m^{j}\right\rangle$ for $j=1,2, \ldots, r$. Taking $r=1$ and $r=2$ in eqn. 6 , we obtain the coupled linear differential equations for the first and second order moments, i.e.,

$$
\frac{d m_{1}}{d t} \simeq-C m_{1}+\frac{A-B}{s}
$$

and

$$
\frac{d m_{2}}{d t} \simeq-2 C m_{2}+\left[\frac{2}{s}(A-B)+C\right] m_{1}+\frac{A+B}{s}
$$

Eqns. $7 a, b$, and $c$, are analogous to those that describe the linear behaviour of the amplifier in the absence of saturation. This can be seen easily by making $s=0$ in eqn. 3. In that case, and for $r=1$ and $r=2$, we obtain

$$
\frac{d m_{1}}{d t}(\text { linear })=(A-B-C) m_{1}+A
$$

and

$$
\begin{aligned}
\frac{d m_{2}}{d t}(\text { linear })= & 2(A-B-C) m_{2} \\
& +(3 A+B+C) m_{1}+A
\end{aligned}
$$

The similarity between eqns. $7 a, b$, and $c$, and $8 a$, and $b$ is obvious, and so we can establish that the behaviour of the strongly saturated optical amplifier is approximately linear.

The solution of the eqns. $7 a, b$, and $c$ is immediate and leads us to

$$
m_{1}(t) \simeq \frac{A-B}{s C}+\left(n_{1}-\frac{A-B}{s C}\right) e^{-C t}
$$

and

$$
\begin{aligned}
m_{2}(t) \simeq & \frac{A-B}{s C} \times\left(\frac{A-B}{s C}+1\right)+\frac{B}{s C} \\
& +\left(\frac{2(A-B)}{s C}+1\right) \\
& \times\left(n_{1}-\frac{A-B}{s C}\right) e^{-C t} \\
& +\left[\left(\frac{A-B}{s C}\right)^{2}-\left(\frac{2(A-B)}{s C}+1\right) n_{1}\right. \\
& \left.+n_{2}-\frac{B}{s C}\right] e^{-2 C t} .
\end{aligned}
$$

where $n_{1}$ and $n_{2}$ are the first and second order moments at the amplifier input $(t=0)$, respectively, and $t$ is the amplification time.

Defining the variance at the amplifier output as

$$
\sigma_{m}^{2}(t)=m_{2}(t)-m_{1}^{2}(t)
$$

and the input one as

$$
\sigma_{n}^{2}=n_{2}-n_{1}^{2}
$$

we obtain the following equations system

$$
\begin{aligned}
m_{1}(t) \simeq & \frac{A-B}{s C}+\left(n_{1}-\frac{A-B}{s C}\right) e^{-C t} \\
\sigma_{m}^{2} \simeq & \frac{A}{s C}+\left(n_{1}-\frac{A-B}{s C}\right) e^{-C t} \\
& +\left(\sigma_{n}^{2}-n_{1}\right) e^{-2 C t}-\frac{B}{s C} e^{-2 C t}
\end{aligned}
$$


Eqns. $12 a$ and $b$ can be studied for different cases. A situation where there is no saturation of the stimulated absorption process would correspond to take $B=0$ in these equations. This behaviour is typical in gas lasers and solid state lasers because they have completely a depopulated lower energy level $\left(n_{s p}=1\right)$. In that case, the expressions of the mean and variance with strong saturation are

$$
\begin{aligned}
& m_{1}(t) \simeq \frac{A}{s C}+\left(n_{1}-\frac{A}{s C}\right) e^{-C t} \\
& \sigma_{m}^{2}(t) \simeq \frac{A}{s C}+\left(n_{1}-\frac{A}{s C}\right) e^{-C t}+\left(\sigma_{n}^{2}-n_{1}\right) e^{-2 C t}
\end{aligned}
$$

and, taking into account eqns. $2 a, b$, and $c$, we obtain

$$
\begin{aligned}
& m_{1}(t) \simeq \frac{1}{s} \times \frac{P}{P_{t h}}+\left(n_{1}-\frac{1}{S} \frac{P}{P_{t h}}\right) e^{-t / z_{p}} \\
& \sigma_{m}^{2}(t) \simeq m_{1}(t)+\left(\sigma_{n}^{2}-n_{1}\right) e^{-2 t / z_{p}}
\end{aligned}
$$

From this set of equations it is easily deduced that if the incident light is coherent $\left(\sigma_{n}^{2}=n_{1}\right)$, the amplified light will also be coherent because $\sigma_{m}^{2} \approx m_{1}$ for any amplification length. If the latter is large enough, the signal-tonoise ratio is approximately equal to $P / s P_{t h}$. This ratio is limited by the condition $m s \gg 1$, but could reach a considerable value if the incident photon number was large.

Nevertheless, in the case of semiconductor amplifiers, the population inversion parameter is considerably greater than unity, and so the mean and variance of the amplified light must be studied starting from eqns. $12 a$ and $b$ which, for the case of coherent light at the amplifier input, take the form

$$
m_{1}(t) \approx \frac{A-B}{s C}+\left(n_{1}-\frac{A-B}{s C}\right) e^{-C t}
$$

and

$$
\sigma_{m}^{2}(t) \approx \frac{A}{s C}+\left(n_{1}-\frac{A-B}{s C}\right) e^{-C t}-\frac{B}{s C} e^{-2 C t}
$$

These latter equations allow us to evaluate, in an analytical way the statistical fluctuations at the output of a strongly saturated amplifier in the presence of coherent light at the input. As an evaluation criterion we can calculate the ratio

$$
F(t)=\frac{m_{1}^{2}(t)}{\sigma_{m}^{2}(t)}
$$

which, for lasers that are long enough, tends towards the value

$$
F_{L}=\frac{(A-B)^{2}}{s A C}
$$

In a general situation, the incident light will not be coherent, owing to the possible quantum noise accumulated before the amplification process that we consider. Under this hypothesis we can assume that

$$
\sigma_{n}^{2}=\alpha n_{1}
$$

being, in general, $\alpha>1$ and $\alpha=1$ for coherent light at the amplifier input (Poisson's statistics). In the next section we will see that, for certain values of the saturation parameter of a semiconductor laser amplifier, it is possible to reduce the relative fluctuations of the amplified light and, in consequence, improve its signal to noise ratio. This ratio presents a maximum for a determinate value of the saturation parameter.

\section{Results and discussion}

Using the method developed in Reference 7, we have calculated the mean, variance and signal-to-noise ratio at the amplifier output as a function of the saturation parameter. These three magnitudes have been plotted with solid lines in Figs. 1, 2 and 3, respec-

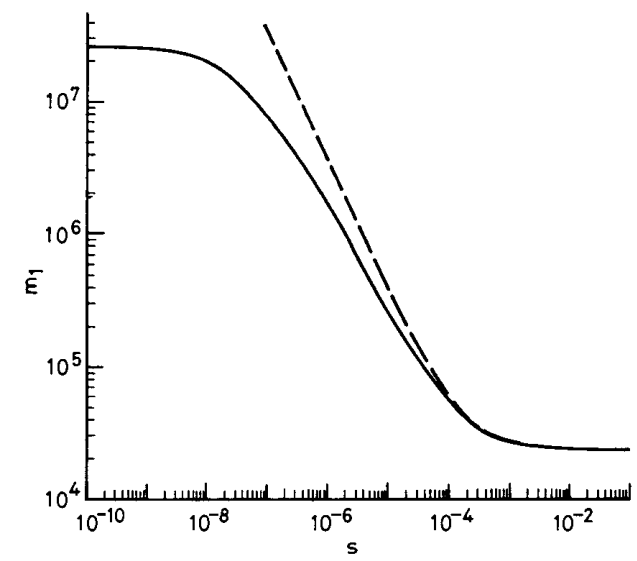

Fig. 1 Plot of $m_{1}$ as a function of the saturation parameter $s$ (solid line). The broken line corresponds to the analytic expression of $m_{1}$ for the case of strong saturation

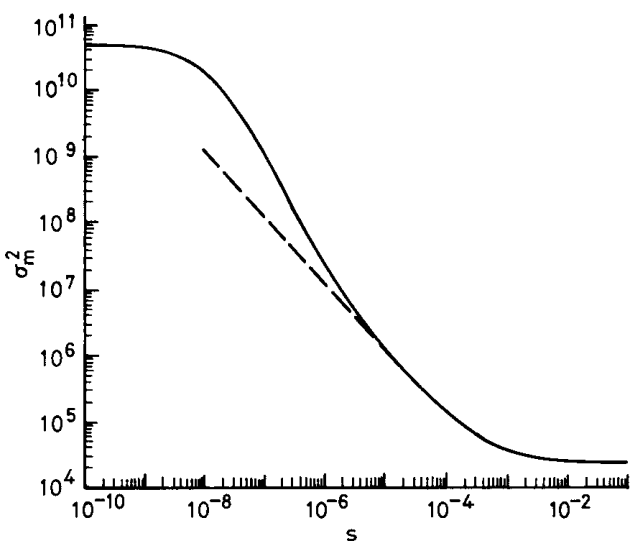

Fig. 2 Plot of $\sigma_{m}^{2}$ as a function of the saturation parameter $s$ (solid line). The broken line corresponds to the analytic expression of $\sigma_{m}^{2}$ for the case of strong saturation

tively, for $A=2.8 \times 10^{12} \mathrm{~s}^{-1}, \quad B=1.82 \times 10^{12} \mathrm{~s}^{-1}$, $C=0.2 \times 10^{12} \mathrm{~s}^{-1}$, and an amplification length of $500 \mu \mathrm{m}$ (the light velocity has been taken to $7 \times 10^{7} \mathrm{~m}$ $\left.s^{-1}\right)$. These values are typical for semiconductor lasers. We have assumed coherent light at the amplifier input with a photon mean number $n_{1}=10^{5}$. For values of $s$ such that $s m \ll 1$ we can consider a linear behaviour of the amplifier and then

$$
\begin{aligned}
m_{1}(t) & \simeq n_{1} e^{(A-B-C) t}+\frac{e^{(A-B-C) t}-1}{A-B-C} A \\
& =n_{1} G_{0}+\frac{G_{0}-1}{A-B-C} A
\end{aligned}
$$

where the equality defines the value $G_{0}$ which represents the unsaturated gain of the amplifier (in this case $G_{0} \approx 24$ 
dB). In Fig. 1 we observe how the gain decreases to one half for $s \approx 3 \times 10^{-8}$. In Fig. 3 we have represented the quotient between the square of the mean and the

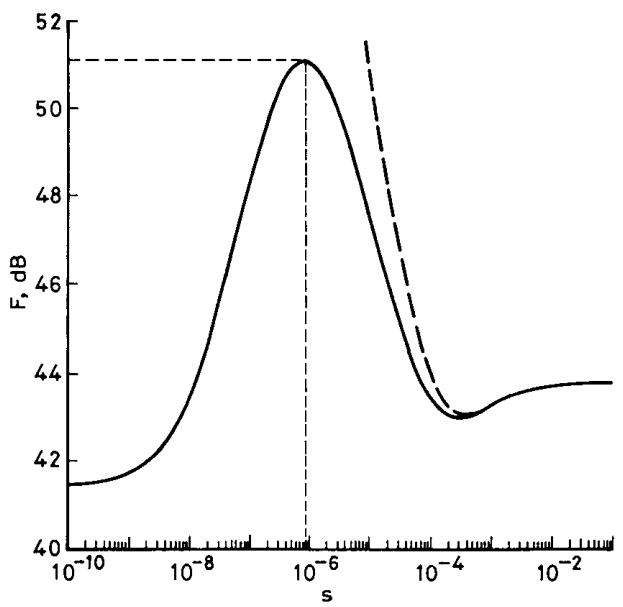

Fig. 3 Plot of the $F$ ratio as a function of the saturation parameter $s$ (solid line). The dashed line corresponds to the analytic expression of the $F$ ratio for the case of strong saturation

variance as a function of the $s$ parameter. It can be observed that the maximum value of this quotient is placed at $s_{M} \approx 10^{-6}$. On the other hand, we have plotted in the same figures eqns. $15 a, 15 b$, and 16 deduced in an analytical way under the condition $s m \gg 1$. For values of the saturation parameter greater than $10^{-4}$, good accordance is observed between the curves obtained analytically and numerically and so, from this value on, an approximately linear behaviour of the amplifier can be considered. In Fig. 4 we represent the $F$ ratio, defined in eqn.

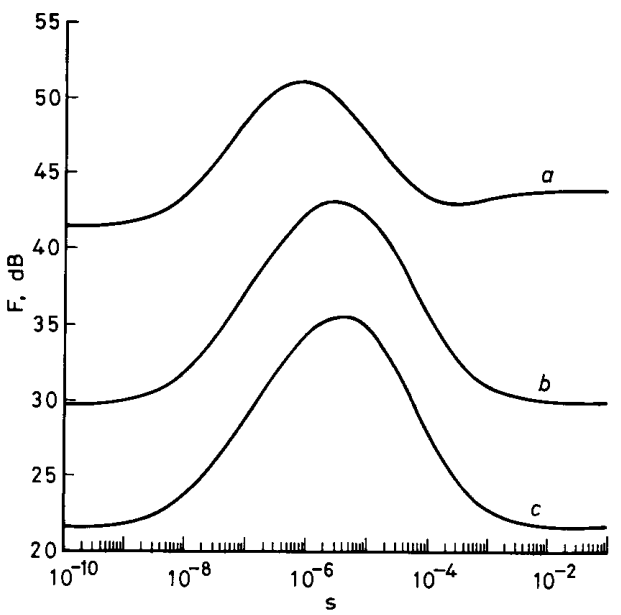

Fig. 4 Plot of the $F$ ratio as a function of the saturation parameter for different values of $\alpha$

curve (a) $\alpha=1$

curve (b) $\alpha=100$

curve (c) $\alpha=640$

16 , as a function of $s$ and taking as a parameter the $\alpha$ coefficient defined in eqn. 18, i.e., taking into account the different situations in which the variance at the amplifier input is greater than the photon mean number. We have taken the same values as in the previous figures for all parameters. It can be seen how the difference between the maximum value of $F$, and its value at the amplifier input $\left(n_{1} / \alpha\right)$ increases with the $\alpha$ coefficient. The case $\alpha=1$ corresponds to the case of coherent light at the amplifier input. If the incident light is less coherent (increase of $\alpha$ ), maximums are obtained for greater values of $s$. Note that for a singal-to-noise ratio of approximately $22 \mathrm{~dB}$ $(\alpha=640)$ at the input, the value of $F$ is slightly greater than $35 \mathrm{~dB}$ for $s=4 \times 10^{-6}$.

\section{Conclusion}

We have presented results describing the nonlinear behaviour of an optical amplifier, taking into account the saturation phenomenon from small values of $s$ up to large values. The effect to consider is the value of the $m s$ term when compared to unity. It is clear that in a situation where saturation is negligible the amplifier is linear. On the other hand, analytical expressions for the mean and variance of the amplified light have been deduced for the case of very-strong saturation. These expressions show that in that situation the device behaves in an approximately linear way. The numerical results presented make us think that it is possible to reduce the relative level of the statistical fluctuations of the incident optical power for certain values of the saturation parameter, and the photon mean number at the amplifier input. For the case of an average photon level at the input of $10^{5}$ (approximately $2 \mathrm{~mW}$ ) with a signal-to-noise ratio of about $22 \mathrm{~dB}(\alpha=640$, Fig. 4 , curve $c)$ an increase of approximately $10 \mathrm{~dB}$ in the output ratio is obtained for a value of the saturation parameter equal to $4 \times 10^{-5}$. This value of $s$ can be considered feasible from the practical point of view $[12,13]$. Nevertheless, the best ratio as has been mentioned in Section 4, is obtained for a value of $s=4 \times 10^{-6}$ which is certainly low although Yamamoto [12], uses a CSP (channelled substrate planar) AlGaAs laser with a value of the saturation parameter $s=5.2 \times 10^{-6}$. These results can be of interest in fibre optic digital transmission systems. Finally, note that the results and conclusions presented in this work have been deduced starting from the statistical behaviour of the optical amplifier. Other characteristics (possible reductions of the bandwidth, influence of the polarisation) must be taken into account in order to evaluate the possible performance introduced by a nonlinear optical amplifier.

\section{References}

1 SIMON, J.C.: 'Semiconductor laser amplifier for single mode optical fiber communications', J. Opt. Comm., 1983, pp. 87-98

2 ADAMS, M.J., COLLINS, J.V., and HENNING, I.D.: 'Analysis of semiconductor laser optical amplifers', IEE Proc. J., 1985, 132, (1), pp. 58-63

3 LOUDON, R.: 'Theory of noise accumulation in linear opticalamplifier chains', IEEE J. Quant. Electron., 1985, QE-21, (7), pp. 766-773

4 YAMAMOTO, Y.: Noise and error rate performance of semiconductor laser amplifers in PCM-IM optical transmission systems, IEEE J. Quant. Electron., 1980, QE-16, (10), pp. 1073-1081

5 MUKAI, T., YAMAMOTO, Y., and KIMURA, T.: 'S/N and error rate performance in AlGaAs semiconductor laser preamplifer and linear repeater systems', IEEE J. Quant. Electron., 1982, QE-18, (10), pp. $1560-1568$

6 RUIZ-MORENO, S., and MORILLO, P.: 'Algebraic techniques application on statistic characterization of an otpical amplifer'. 5th International Conference on Applied algebra, algebraic algorithms and error-correcting-codes. Menorca, Spain, 1987

7 RUIZ-MORENO, S., JUNYENT, G., USANDIZAGA, J.R., and CALZADA, A.: 'Resolution of the moment equations in a nonlinear optical amplifier', Electron. Lett., 1987, 23, No. 1, pp. 15-17

8 YAMAMOTO, Y.: 'AM and FM quantum noise in semiconductor lasers - Part I: Theoretical analysis', IEEE J. Quant. Electron. 1983, QE-19, (1), pp. 34-46 
9 SHIMODA, K., TAKAHASI, I., and TOWNES, C.H.: 'Fluctuations in amplification of quanta with application to maser amplifiers', J. Phys. Soc. Japan, 1957, 12, pp. 686-700

10 BENDJABALLAH, C., and OLIVER, G.: 'Detection of coherent light after nonlinear amplification', IEEE Trans. on Aerosp. and Elect. Systems, 1981, AES-17, No. 5, pp. 620-625

11 MUKAI, T., YAMAMOTO, Y., and KIMURA, T.: 'Optical direct amplification for fiber transmission', Review of Elec. Commun. Laboratories, 1983, 31, (3), pp. 340-348

12 YAMAMOTO, Y.: 'AM and FM quantum noise in semiconductor lasers - Part II: Comparison of theoretical and experimental results for AlGaAs lasers', IEEE J. Quantum. Electron., 1983, QE-19, (1), pp. 47-58

13 SHARFIN, W.F., and DAGENAIS, M.: 'Dynamics of optically switched bistable diode laser amplifiers', IEEE J. Quantum. Electron., 1987, QE-23, (3), pp. 303-308

14 OLIVER, G., and BENDJABALLAH, CH.: 'Statistical properties of coherent radiation in a nonlinear optical amplifier', Phys. Rev. A, $1980,22,(2)$, pp. $630-634$

15 BENDJABALLAH, CH., and OLIVER, G.: 'Comparison of statistical properties of two models for saturated laser-light amplifier', Phys. Rev. A, 1980, 22, (6), pp. 2726-2731

\section{Erratum}

BAILLIE, W.L.: 'Developments of reflection mode liquid crystal light values using $\mathrm{Bi}_{12} \mathrm{SiO}_{20}$ as the photoconductor', IEE Proc. J, Optoelectron., 1987, 134, (6), pp. 326-332

Eqn. 3 should read

$$
\text { O.M. }=\frac{\left(I_{0} I_{1}\right)^{1 / 2}}{\frac{1}{4} I_{0}+I_{1}}
$$

